\title{
Lumbar spinal stenosis associated with progression of locomotive syndrome and lower extremity muscle weakness
}

This article was published in the following Dove Press journal:

Clinical Interventions in Aging

\section{Yuji Kasukawa \\ Naohisa Miyakoshi \\ Michio Hongo \\ Yoshinori Ishikawa \\ Daisuke Kudo \\ Hiroaki Kijima \\ Ryota Kimura \\ Yuichi Ono \\ Yasuhiro Takahashi \\ Yoichi Shimada}

Department of Orthopedic Surgery, Akita University Graduate School of Medicine, Akita, Japan
Correspondence: Yuji Kasukawa Department of Orthopedic Surgery, Akita University Graduate School of Medicine, I-I-I Hondo, Akita 010-8543, Japan

Tel +81188846148

Fax +81188362617

Email kasukawa@doc.med.akita-u.ac.jp
Purpose: The purpose of this study was to evaluate the association between the early stages of lumbar spinal stenosis (LSS) and the risk of locomotive syndrome, as well as its effect upon muscle strength of the back, upper extremities, and lower extremities.

Patients and methods: LSS was diagnosed with a self-administered, self-reported history questionnaire. Participants $(n=113)$ who agreed to be tested by the diagnostic support tool for LSS underwent three risk tests for locomotive syndrome: a stand-up test, a two-step test, and a 25-question Geriatric Locomotive Function Scale (GLFS-25), as well as measurements of the strength of their grip, back extensor, hip flexor, and knee extensor muscles.

Results: Twenty-three participants were diagnosed with LSS by the questionnaire. Results of the stand-up test in the LSS group were significantly worse than those in the no-LSS group $(P=0.003)$. The results of the two-step test and the total score on the GLFS-25 in the LSS group were significantly worse than those in the no-LSS group ( $P=0.002$ and $P<0.0001$, respectively). The stages of locomotive syndrome assessed by the stand-up test, two-step test, and the GLFS-25 were significantly worse in the LSS group than in the no-LSS group $(P=0.0004, P=0.0007$, and $P<0.0001$, respectively). Hip flexor and knee extensor strength, but not grip and back extensor strength, in the LSS group were significantly lower than that in the no-LSS group.

Conclusions: LSS diagnosed using the self-reported support tool worsened the stage of locomotive syndrome in older people. Furthermore, participants with LSS had significant lower extremity weakness.

Keywords: lumbar spinal stenosis, locomotive syndrome, locomotive syndrome risk test, grip strength, back extensor strength, lower extremity muscle strength

\section{Short summary}

The early stages of LSS diagnosed using the self-reported support tool worsen the stage of locomotive syndrome by the stand-up test, two-step test, and the 25-question Geriatric Locomotive Function Scale in older people. Furthermore, participants with LSS had significant lower extremity weakness, but not grip strength nor back extensor strength.

\section{Introduction}

The percentage of people $>65$ years is rapidly increasing in Japan, and comprised $27.7 \%$ of the entire population in $2017 .{ }^{1}$ A cause of health disparity in this population is impairment in locomotive function, which is associated with an increased risk for needing nursing support. Independence in activities of daily living (ADL) and extension of a healthy life expectancy are important social values in an aging society. 
Locomotive syndrome is a condition proposed by the Japanese Orthopaedic Association (JOA) in 2007 to increase societal awareness regarding impairment of locomotive function of elderly people and its management strategies. ${ }^{2,3}$ The means and purpose of management of locomotive syndrome are understood and accepted by the general population. ${ }^{2,3}$ It is a condition wherein mobility functions such as sit-to-stand and gait have declined because of locomotive organ disorders. The JOA introduced a battery of short tests: the "standup test", "two-step test", 5 and a 25-question Geriatric Locomotive Function Scale (GLFS-25) ${ }^{6,7}$ for the early detection of locomotive syndrome and its attendant risks. ${ }^{2,3}$

Lumbar spinal stenosis (LSS) is one of the risk factors for locomotive syndrome. ${ }^{3}$ LSS is a degenerative condition that causes intermittent neurogenic claudication. Several previous studies have reported that LSS and its severity are associated with locomotive syndrome ${ }^{8}$ and its progression. ${ }^{9}$ However, the process by which early-stage LSS affects the risk for locomotive syndrome is still unknown. To detect early-stage LSS, for which surgical treatment is not indicated, we used a diagnostic support tool for LSS. ${ }^{10}$ The diagnostic support tool for LSS was reported in a retrospectively performed derivation study of 137 patients' symptoms, physical examination, and magnetic resonance imaging (MRI) findings in the previous study. ${ }^{11}$ The tool comprises a simple, self-administered, and self-reported history questionnaire that is useful in the diagnosis of LSS with high sensitivity $(92.7 \%)$ and specificity $(84.7 \%)$ and does not require physical or radiological examinations. ${ }^{10}$ Our goal was to evaluate the association between early-stage LSS diagnosed with the diagnostic support tool and the risk level for locomotive syndrome as well as the association between the early stage of LSS and muscle strength of the back, upper extremities, and lower extremities.

\section{Methods}

\section{Patients and study design}

The participants were selected from people undergoing medical checkups at municipal health centers between 2016 and 2017. After receiving an explanation of the locomotive syndrome risk tests and the measurements of muscle strength, all subjects provided written informed consent prior to participation. Inclusion criteria were all subjects aged over 50 years, and who agreed to take the examination. Exclusion criteria were subjects who did not agree to take the examination, could not walk without any support, or who had any pain or diseases that would have made measurement difficult. All participants who answered the LSS diagnostic support tool underwent the three risk tests for locomotive syndrome, and they were tested for muscle strength. The study protocol was approved by the ethics committee of the Akita University Graduate School of Medicine (IRB \#669). This study was conducted in accordance with the Declaration of Helsinki.

\section{Diagnosis of LSS}

LSS was diagnosed using the diagnostic support tool described by Aizawa et al. ${ }^{10}$ The tool is a self-administered,

Table I LSS diagnostic support tool

\begin{tabular}{|l|l|l|}
\hline & Question & LSS score \\
\hline Q1 & Numbness and/or pain in the thigh(s) down to the calf (calves) and shin(s) & N.A. \\
Q2 & Difficulty bending forward because of numbness and/or pain & - I \\
Q3 & Difficulty putting on socks because of numbness and/or pain & - I \\
Q4 & Difficulty lying on the back with hip and knee extension because of numbness and/or pain & N.A. \\
Q5 & Difficulty sitting with knee extension of the involved leg & N.A. \\
Q6 & Numbness and/or pain increase in intensity after walking for a while, but are relieved by taking a rest & 5 \\
Q7 & Standing for a while brings numbness and/or pain in the thigh(s) down to the calf or calves and shin(s) \\
Q8 & Numbness and/or pain are reduced by bending forward & 5 \\
Q9 & Numbness is present, but pain is absent & I \\
Q10 & Numbness and/or pain is present in both feet & I \\
Q11 & Numbness is present in soles of both feet & 3 \\
Q12 & Numbness arises around the buttocks & 3 \\
Q13 & Feel the urge to urinate when walking & N.A. \\
Q14 & I am $\leq 40$ years old & N.A. \\
Q15 & I am $\geq 60$ years old & 4 \\
\hline
\end{tabular}

Notes: Adapted from Aizawa T, Tanaka Y, Yokoyama T, et al. New diagnostic support tool for patients with leg symptoms caused by lumbar spinal stenosis and lumbar intervertebral disc herniation: a self-administered, self-reported history questionnaire. J Orthop Sci. 2016;2I(5):579-585. ${ }^{10}$ Copyright 2016, with permission from Elsevier. Abbreviations: LSS, lumbar spinal stenosis; N.A., not applicable; Q, question number. 
self-reported history questionnaire containing 15 "Yes" or "No" questions (Table 1). Ten questions relate to LSS (\#2, 3, $6,7,8,9,10,11,12$, and 15) and are weighted (scored from -1 to 5). Participants with a total score $>13$ were considered to have LSS. The remaining questions (\#1, 4, 5, 13, and 14) relate to lumbar disc herniation, which we did not address in this study. The sensitivity and specificity of this tool for the diagnosis of LSS have been reported as $92.7 \%$ and $84.7 \%$, respectively. ${ }^{10}$

\section{Locomotive syndrome risk testing}

Three tests for locomotive syndrome were performed according to JOA guidelines. ${ }^{3}$

In the stand-up test, the ability to stand up with a single- or double-leg stance from stools that were 40, 30, 20 , and $10 \mathrm{~cm}$ in height was evaluated. The difficulty was graded from $40 \mathrm{~cm}$ both legs, $<30 \mathrm{~cm}$ both legs, $<20 \mathrm{~cm}$ both legs, $<10 \mathrm{~cm}$ both legs, $<40 \mathrm{~cm}$ one leg, $<30 \mathrm{~cm}$ one leg, $<20 \mathrm{~cm}$ one leg, to $10 \mathrm{~cm}$ one leg. The result was recorded as the minimum stool height from which the participant could stand up from. ${ }^{4}$ The minimum stool height of $10 \mathrm{~cm}$ or $30 \mathrm{~cm}$ with both legs was graded as stage 1 or stage 2 , respectively. ${ }^{3}$

In the two-step test, the length of two strides from a starting line was measured. The score was calculated by normalizing the maximal length of two steps by the height of participants. ${ }^{5}$ Those with scores $<1.3$ or $<1.1$ were defined as stage 1 or stage 2 , respectively. ${ }^{3}$

The GLFS-25 is a self-reported comprehensive questionnaire about activities of the preceding month. ${ }^{6,7}$ The scale includes four questions regarding pain, 16 questions regarding ADL, three questions regarding social functions, and two questions regarding mental health status. Each question was graded from no impairment ( 0 points $)$ to severe impairment ( 4 points). Those with total scores $\geq 7$ or $\geq 16$ were graded as stage 1 or stage 2 , respectively. ${ }^{3}$

\section{Muscle strength (Figure I)}

Grip strength was measured on the dominant and nondominant sides using a TTM dynamometer (Tsutsumi, Tokyo, Japan), with the participant in the standing position. The test was performed twice, and the mean of the right and left sides was determined. Muscle strength of the lower extremities, including hip flexors and knee extensors, was measured using the JTECH Commander PowerTrack II handheld dynamometer (Nihon Medix Co., Ltd., Chiba, Japan) (Figure $1 \mathrm{~A}$ and $\mathrm{B})$. The strength of the muscle groups of the lower extremities was measured twice and the mean of the bilateral
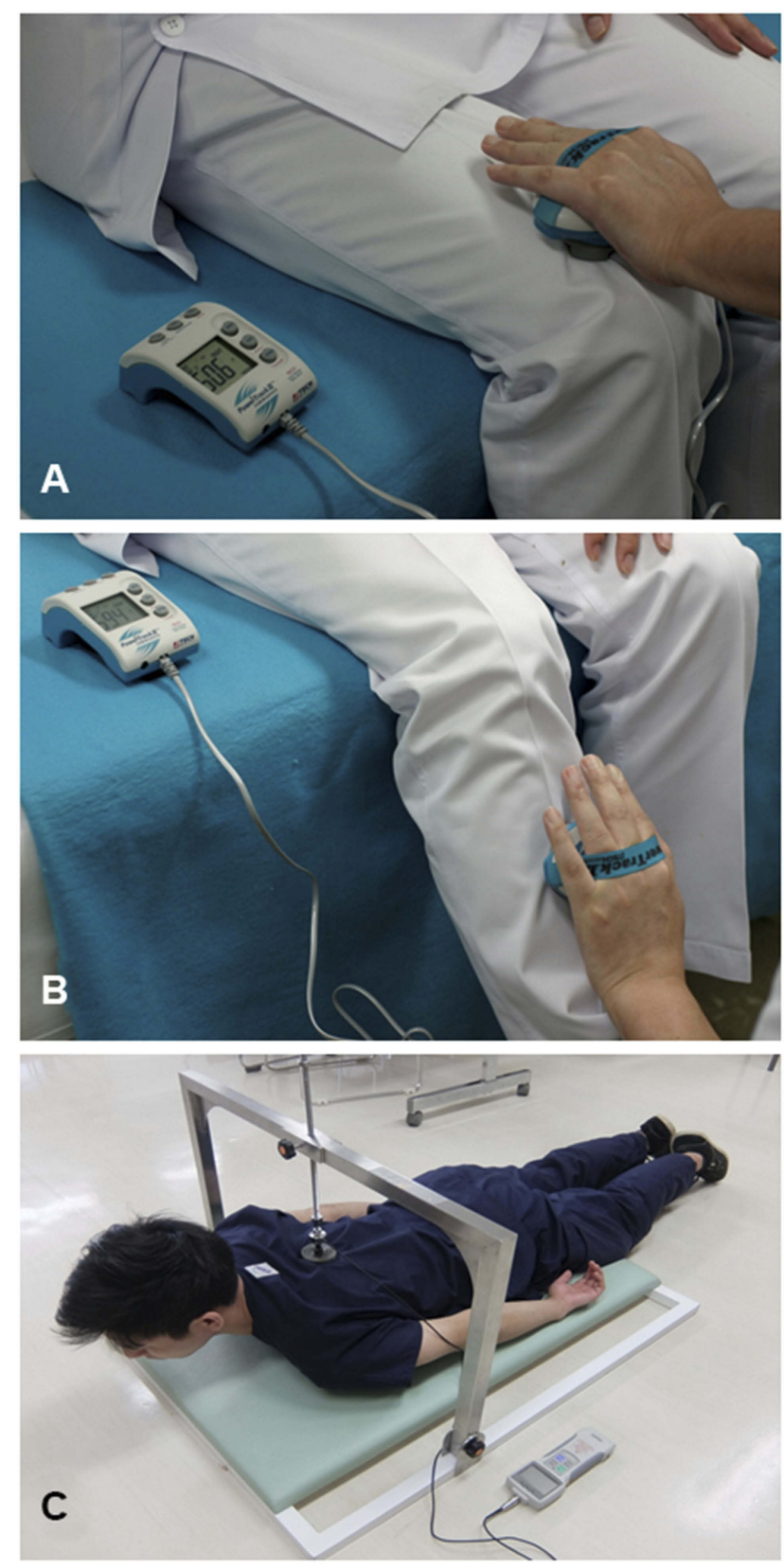

Figure I The muscle strength of the lower extremities, including hip flexors (A) and knee extensors (B), was measured with a handheld dynamometer (Commander ${ }^{\mathrm{TM}}$ PowerTrack II ${ }^{\mathrm{TM}}$ Handheld Dynamometer; Nihon Medix Co., Ltd., Matsudo, Japan). The mean strength of the two lower extremities was calculated and all calculations were performed twice. Isometric back extensor strength in the prone position was measured using a strain-gauge dynamometer (DPU-I000N Digital Force Gauge; Imada, Toyohashi, Japan) (C). The subject was aligned on the board with a strain-gauge dynamometer attached to the subject's back. After the strain-gauge was placed (at the mid-portion of back at the inferior part of scapula), we asked the subject to lift their upper trunk as high as possible. The strain-gauge recorded the maximum strength. Measurements were performed three times and the values from the trial that produced the greatest force were selected.

measurements was recorded. ${ }^{12}$ Isometric back extensor strength was measured using a digital force strain-gauge dynamometer (Imada Co., Ltd., Toyohashi, Japan). To obtain these measurements, the participant lay prone on the table, and 
the strain-gauge dynamometer was placed in the mid portion (at the inferior aspect of the scapula) of the back. The participant was then asked to lift the upper trunk as much as possible (Figure 1C). Maximum strength was then determined using the strain gauge. Measurements were performed three times and the maximum value was recorded, as previously described. ${ }^{13}$ The coefficient of variation, as an indicator of the precision of the measurement, was $2.3 \%{ }^{14}$

\section{Statistical analyses}

The results were expressed as the mean ( \pm standard deviation) for parametric data or median [lower, upper quartiles] for nonparametric data. A Kolmogorov-Smirnov test revealed normal distributions of the data except LSS score, two-step test, and GLFS-25. The differences in data between the LSS and no-LSS groups were assessed using the unpaired $t$-test, without nonparametric data such as LSS score, two-step test, and GLFS-25, which were assessed by the Mann-Whitney $U$ test. Differences between the groups were analyzed using the chi-squared test or Fisher's exact test for nominal variables such as gender or stand-up test and risk levels for locomotive syndrome (Stages 0, 1, and 2). Differences with a $P$-value $<0.05$ were considered statistically significant. All statistical analyses were performed using EZR (Easy R) statistical software (The R Foundation for Statistical Computing). ${ }^{15}$

\section{Results}

\section{Participant characteristics}

Table 2 presents the characteristics of the participants. Twenty-three participants were diagnosed with LSS by the LSS score $(\geq 13)$ on the questionnaire. The median LSS scores of the LSS and no-LSS groups were 17 and 5.3 , respectively. There was no significant difference in gender percentage: 15 males and 8 females in the LSS group, and 43 males and 47 females in the no-LSS group.

Table 2 Participant characteristics

\begin{tabular}{|l|l|l|l|}
\hline & LSS+ & LSS- & P-value \\
\hline Number of participants & 23 & 90 & \\
LSS score & $17.0[14.0,18.5]$ & $5.3[4.0,5.0]$ & $<0.00 I^{*}$ \\
Male:female & $15: 8$ & $43: 47$ & $0.164^{\#}$ \\
Average age, years & $75.9(9.4)$ & $73.5(7.8)$ & $0.226^{* *}$ \\
Height, cm & $158.2(7.3)$ & $155.4(8.0)$ & $0.134^{* *}$ \\
Weight, kg & $59.6(10.4)$ & $55.1(10.1)$ & $0.060^{* *}$ \\
\hline
\end{tabular}

Notes: Mean (standard deviation), Median [lower, upper quartiles]. Cut-off score for LSS diagnosis: LSS+, $\geq 13$; LSS-, <13. *Mann-Whitney U-test; ${ }^{\#}$ Chi-square test; **Unpaired $t$-test.

Abbreviation: LSS, lumbar spinal stenosis.
Mean age, height, and weight did not show a significant difference between the groups.

\section{Stand-up test, two-step test, and GLSF-25 (Table 3)}

Forty-three percent of participants in the LSS group stood up from the $10 \mathrm{~cm}$ height with both legs, and $43 \%$ of participants in the no-LSS group stood up from the $40 \mathrm{~cm}$ height with one leg. The stand-up test in the LSS group was significantly worse than that in the no-LSS group $(P=0.003)$. The value from the two-step test and the total score from the GLFS-25 in the LSS group (1.21 and 21.7, respectively) were significantly worse than those in the no-LSS group (1.36 and 5.0, respectively) $(P=0.002$ and $P<0.0001$, respectively). Subanalyses showed that the GLFS-25 scores related to pain, ADL, social functions, and mental health questions in the LSS group $(5.9,10.5,3.1$, and 2.2, respectively) were significantly worse than those in the no-LSS group $(1.9,1.8$, 0.7 , and 0.6 , respectively) $(P<0.0001)$.

\section{Locomotive syndrome risk test staging (Table 4)}

The percentage of subjects with locomotive syndrome stage worsening by the stand-up test in the LSS group was: Stage 0 , 7; Stage 1, 12; and Stage 2, 4 . These results were significantly higher $(P=0.0004)$ than those in the no-LSS group (Stage 0 , 54; Stage 1, 36; and Stage 2, 0). Locomotive syndrome stage worsening by the two-step test was also significantly greater in the LSS group (Stage 0, 9; Stage 1, 6; and Stage 2,8) than in the no-LSS group (Stage 0, 66; Stage 1, 11; and Stage 2, 13) $(P=0.0007)$. Similar results were noted for the GLFS-25, with significant worsening in the LSS group (Stage 0, 2; Stage 1, 9; and Stage 2, 12) than in the no-LSS group (Stage 0, 60; Stage 1, 24; and Stage 2, 6) $(P<0.0001)$.

\section{Muscle strength (Table 5)}

Grip strength and back extensor strength were not significantly different between the LSS and no-LSS groups. Hip flexor strength $(10.6 \mathrm{~kg})$ and knee extensor strength (11.5 $\mathrm{kg}$ ) in the LSS group were significantly lower than those (13.4 kg and $13.6 \mathrm{~kg}$, respectively) in the no-LSS group ( $P=0.014$ and $P=0.017$, respectively).

\section{Discussion}

\section{Locomotive function in super-aging societies}

It is predicted that the proportion of the population aged $\geq 65$ years in Japan will reach $38.4 \%$ by $2065 .{ }^{1}$ The aging 
Table 3 Locomotive syndrome risk test

\begin{tabular}{|c|c|c|c|}
\hline & LSS+ $(n=23)$ & LSS- $(n=90)$ & $P$-value \\
\hline Stand-up test & & & $0.003^{\# \#}$ \\
\hline Bilateral legs $40 \mathrm{~cm}$ & 0 & 0 & \\
\hline 30 cm (\%) & $4(17)$ & 0 & \\
\hline $20 \mathrm{~cm} \mathrm{( \% )}$ & $2(9)$ & $10(12)$ & \\
\hline 10 cm (\%) & $10(43)$ & $26(29)$ & \\
\hline One leg 40 cm (\%) & $7(31)$ & $39(43)$ & \\
\hline 30 cm (\%) & 0 & $12(13)$ & \\
\hline $20 \mathrm{~cm}(\%)$ & 0 & I (I) & \\
\hline $10 \mathrm{~cm}(\%)$ & 0 & $2(2)$ & \\
\hline Two-step test & $1.21[1.06,1.38]$ & $1.36[1.25,1.49]$ & $0.002 *$ \\
\hline \multicolumn{4}{|l|}{ GLFS-25 } \\
\hline Total score & $21.7[13.5,28.5]$ & $5.0[2.0,7.0]$ & $<0.000 I^{*}$ \\
\hline Pain-related score & $5.9[3.5,8.5]$ & $1.9[0.0,3.0]$ & $<0.000 I^{*}$ \\
\hline ADL-related score & $10.5[3.5,15.5]$ & $1.8[0.0,3.0]$ & $<0.0001 *$ \\
\hline Social-functions-related score & $3.1[1.0,4.0]$ & $0.7[0.0,1.0]$ & $<0.0001 *$ \\
\hline Mental-health-related score & $2.2[1.0,4.0]$ & $0.6[0.0,1.0]$ & $<0.0001 *$ \\
\hline
\end{tabular}

Notes: Median [lower, upper quartiles]. ${ }^{\#}$ Fisher's exact test; *Mann-Whitney U-test; Cut-off score for LSS diagnosis: LSS+, $\geq 13$; LSS-, <I3.

Abbreviations: ADL, activities of daily living; LSS, lumbar spinal stenosis; GLFS-25, 25-question Geriatric Locomotive Function Scale.

population ratio is also rapidly increasing worldwide including in the United States, Europe, China, South Korea, and other Asian countries. ${ }^{16}$ In super-aging societies, it is very important that older people maintain their locomotive function. In our study, the subjects diagnosed with early-stage LSS by the diagnostic support tool were at higher risk of locomotive syndrome than the people without LSS. The participants with LSS had comparatively decreased strength in the lower extremities, but not in the grip or back extensor muscles, compared with

Table 4 Risk levels for locomotive syndrome from the three tests

\begin{tabular}{|c|l|l|l|}
\hline & LSS+ (n=23) & LSS $-(\mathbf{n = 9 0 )}$ & P-value $^{\#}$ \\
\hline Stand-up test & & & 0.0004 \\
Stage 0 (\%) & $7(30)$ & $54(60)$ & \\
Stage I (\%) & $12(52)$ & $36(40)$ & \\
Stage 2 (\%) & $4(18)$ & $0(0)$ & \\
\hline Two-step test & & & 0.0007 \\
Stage 0 (\%) & $9(39)$ & $66(73)$ & \\
Stage I (\%) & $6(26)$ & $11(12)$ & \\
Stage 2 (\%) & $8(35)$ & $13(15)$ & $<0.000 I$ \\
\hline GLFS-25 & & & \\
Stage 0 (\%) & $2(9)$ & $60(67)$ & \\
Stage I (\%) & $9(39)$ & $24(27)$ & \\
Stage 2 (\%) & $12(52)$ & $6(6)$ & \\
\hline
\end{tabular}

Notes: ${ }^{\#}$ Fisher's exact test. Cut-off score for LSS diagnosis: LSS,$+ \geq 13$; LSS-, $<13$. Abbreviations: LSS, lumbar spinal stenosis; GLFS-25, 25-question Geriatric Locomotive Function Scale. those without LSS. We speculate that the decreased strength in the lower extremities caused the locomotive syndrome worsening in the subjects in the early stages of LSS.

\section{LSS and locomotive syndrome and risk of locomotive syndrome}

It has been reported that low back pain, hip pain, and knee pain are significantly associated with locomotive dysfunction. ${ }^{17}$ In addition to musculoskeletal pain, several recent studies have indicated that LSS also causes the worsening of locomotive syndrome. ${ }^{11,18}$ The prevalence of LSS identified by another diagnostic support tool correlated with worse results in the GLFS-25. ${ }^{8}$ To the best of our knowledge, this is the first study to demonstrate the association of LSS with not only the GLFS-25 score but also with the stand-up test and the two-step test scores for evaluation of locomotive syndrome severity. Fujita et al reported that patients with LSS, who were treated with surgery, were diagnosed as having locomotive syndrome, and the LSS severity was associated with the degree of locomotive syndrome. ${ }^{9}$ The subjects with LSS in our study were diagnosed using the self-reported questionnaire, and were at risk for locomotive syndrome even though they were in the relatively early stages of LSS and surgery was not indicated. Based on the results of this study, we consider that early interventions for LSS, such as medical treatment or patient 
Table 5 Muscle strength

\begin{tabular}{|l|l|l|l|}
\hline & $\begin{array}{l}\text { LSS+ } \\
(\mathbf{n = 2 3 )}\end{array}$ & $\begin{array}{l}\text { LSS- } \\
(\mathbf{n = 9 0 )}\end{array}$ & $\begin{array}{l}\boldsymbol{P} \text { - } \\
\text { value** }\end{array}$ \\
\hline Grip strength, kg & $27.9(8.8)$ & $28.2(8.6)$ & 0.905 \\
Back extensor strength, kg & $16.1(10.0)$ & $19.9(10.5)$ & 0.128 \\
Hip flexor strength, kg & $10.6(4.7)$ & $13.4(4.9)$ & 0.014 \\
Knee extensor strength, kg & $11.5(3.6)$ & $13.6(3.7)$ & 0.017 \\
\hline
\end{tabular}

Notes: Mean (standard deviation). **Unpaired $t$-test; Cut-off score for LSS diagnosis: LSS+, $\geq 13$; LSS-, <13.

Abbreviation: LSS, lumbar spinal stenosis.

education, might reduce the risk of locomotive syndrome and might prevent its worsening.

\section{LSS and muscle strength of the back extensors and lower extremities}

The participants with LSS diagnosed by the support tool showed decreased strength in the lower extremities (hip flexors and knee extensors). The diagnostic support tool for LSS used in this study includes questions on the prevalence of pain and numbness during walking or standing. Another study, which used a different diagnostic support tool that included a question on numbness of the soles at rest, reported that sole numbness was associated with lower scores for quality of life and ADL. ${ }^{19}$ We speculate that the weakened muscle strength of the lower extremities as well as the presence of numbness or pain in the participants' buttocks, legs, and soles caused the worse results in the stand-up and two-step tests.

\section{LSS and pain, social function, and mental health}

Results of the subanalyses of the GLFS-25 showed that the subjects with early-stage LSS had significantly worse scores related to pain, social function, and mental health. Several previous studies have demonstrated that LSS causes impairment of health-related quality of life (HRQOL). Shigematsu et al reported that patients with LSS had a lower HRQOL and more of them had locomotive syndrome than older persons without LSS. ${ }^{20}$ Backpain-related disability and HRQOL were reported to be significantly associated with some physical fitness parameters in patients with LSS. ${ }^{21}$ These results and our study indicate that LSS causes physical and also HRQOL impairment including pain and mental health issues. Treatment for LSS results in significant improvement in physical and psychological status as well as improvement in HRQOL. Haro et al reported that surgical treatment for LSS improved mental health, as evaluated by the mental component summary score of the Short-Form 36 Health Survey. ${ }^{22}$ Surgical intervention showed greater postoperative improvement in pain severity, functional impairment, and HRQOL in younger patients than in older patients. ${ }^{23}$ Thus, early detection of LSS and locomotive syndrome using a self-reported diagnostic support tool and locomotive syndrome risk assessment followed by early treatment for LSS should be important in maintaining independence in ADL and extending life expectancy in older people.

\section{Study limitations}

There are several limitations to this study. First, the diagnosis of LSS was made using only a self-reported diagnostic support tool. There was no evaluation of neurological deficits by physical examination, and no other diagnostic studies for LSS such as MRI or other radiological examinations were performed. However, use of the support tool in this study was useful in detecting the early stages of LSS. Second, the number of subjects in the two groups was different, which might have introduced statistical weakness. Third, the presence or absence of knee or hip osteoarthritis was not evaluated using X-ray in this study. Osteoarthritis of the knee or hip joint exerts influence on locomotive function in older people.

\section{Conclusions}

The older subjects with LSS diagnosed using the selfreported support tool exhibited locomotive syndrome worsening as evaluated by the stand-up test, two-step test, and GLFS-25. Those participants with LSS had weakened lower extremity muscle strength.

\section{Acknowledgments}

The authors thank doctors Hiroyuki Nagasawa, Koji Nozaka, Hidetomo Saito, Hiroyuki Tsuchie, Kimio Saito, Masashi Fujii, Manabu Akagawa, Jumpei Iida, Itsuki Nagahata, Yusuke Yuasa, Yosuke Iwamoto, Hiroaki Tsukamoto, Chiaki Sato, and Junichi Inoue for performing the locomotive syndrome risk tests and the measurement of muscle strengths. The authors thank Eriko Kamada and Maki Takeshita for their help in the data analysis. We also thank medical students for helping with the measurements for the locomotive syndrome risk test. We thank Andrea Baird, MD, and Libby Cone, MD, MA, from Edanz Group for editing drafts of this manuscript. 


\section{Disclosure}

The authors report no conflicts of interest in regard to this work.

\section{References}

1. The state of the aging population. In: White Paper on the Aging Society. Cabinet Office, Government of Japan; 2017. Available from: https://www8.cao.go.jp/kourei/whitepaper/w-2018/html/ gaiyou/s11.html.

2. Nakamura K. The concept and treatment of locomotive syndrome: its acceptance and spread in Japan. J Orthop Sci. 2011;16(5):489-491. doi:10.1007/s00776-011-0108-5

3. Nakamura K, Ogata T. Locomotive syndrome: definition and management. Clin Rev Bone Miner Metab. 2016;14(2):56-67. doi:10.1007/s12018-016-9208-2

4. Muranaga S. Evaluation of the muscular strength of the lower extremities using the standing movement and clinical application. J Showa Med Assoc. 2001;61(3):362-367.

5. Muranaga S, Hirano K. Development of a convenient way to predict ability to walk, using a two-step test. J Showa Med Assoc. 2003;63 (3):301-303.

6. Seichi A, Hoshino Y, Doi T, Akai M, Tobimatsu Y, Iwaya T. Development of a screening tool for risk of locomotive syndrome in the elderly: the 25-question geriatric locomotive function scale. $J$ Orthop Sci. 2012;17(2):163-172. doi:10.1007/s00776-011-0193-5

7. Ogata T, Muranaga S, Ishibashi H, et al. Development of a screening program to assess motor function in the adult population: a crosssectional observational study. J Orthop Sci. 2015;20(5):888-895. doi:10.1007/s00776-015-0737-1

8. Chiba D, Tsuda E, Wada K, et al. Lumbar spondylosis, lumbar spinal stenosis, knee pain, back muscle strength are associated with the locomotive syndrome: rural population study in Japan. $J$ Orthop Sci. 2016;21(3):366-372. doi:10.1016/j.jos.2016.02.006

9. Fujita N, Sakurai A, Miyamoto A, et al. Lumbar spinal canal stenosis leads to locomotive syndrome in elderly patients. J Orthop Sci. 2018; pii: S0949-2658(18)30228-8. doi:10.1016/j.jos.2018.08.004.

10. Aizawa T, Tanaka Y, Yokoyama T, et al. New diagnostic support tool for patients with leg symptoms caused by lumbar spinal stenosis and lumbar intervertebral disc herniation: a self-administered, selfreported history questionnaire. J Orthop Sci. 2016;21(5):579-585. doi:10.1016/j.jos.2016.07.012

11. Konno S, Kikuchi S, Tanaka Y, et al. A diagnostic support tool for lumbar spinal stenosis: a self-administered, self-reported history questionnaire. BMC Musculoskelet Disord. 2007;30(8):102. doi:10.1186/1471-2474-8-102

12. Kasukawa Y, Miyakoshi N, Hongo M, et al. Age-related changes in muscle strength and spinal kyphosis angles in an elderly Japanese population. Clin Interv Aging. 2017;20(12):413-420. doi:10.2147/ CIA.S113352

Clinical Interventions in Aging

\section{Publish your work in this journal}

Clinical Interventions in Aging is an international, peer-reviewed journal focusing on evidence-based reports on the value or lack thereof of treatments intended to prevent or delay the onset of maladaptive correlates of aging in human beings. This journal is indexed on PubMed Central, MedLine, CAS, Scopus and the Elsevier
13. Miyakoshi N, Hongo M, Maekawa S, et al. Factors related to spinal mobility in patients with postmenopausal osteoporosis. Osteoporos Int. 2005;16(12):1871-1874. doi:10.1007/s00198-0051953-x

14. Miyakoshi N, Hongo M, Maekawa S, Ishikawa Y, Shimada Y, Itoi E. Back extensor strength and lumbar spinal mobility are predictors of quality of life in patients with postmenopausal osteoporosis. Osteoporos Int. 2007;18(10):1397-1403. doi:10.1007/s00198-0070383-3

15. Kanda Y. Investigation of the freely available easy-to-use software 'EZR' for medical statistics. Bone Marrow Transplant. 2013;48 (3):452-458. doi:10.1038/bmt.2012.244

16. United Nations. World Population Prospects -2017 Revision: Global population. Available from: https://www.un.org/development/desa/ publications/graphic/wpp2017-global-population. Accessed May 5, 2019.

17. Iizuka Y, Iizuka H, Mieda T, Tajika T, Yamamoto A, Takagishi K. Population-based study of the association of osteoporosis and chronic musculoskeletal pain and locomotive syndrome: the Katashina study. $J$ Orthop Sci. 2015;20(6):1085-1089. doi:10.1007/s00776-015-0774-9

18. Yabuki S, Fukumori N, Takegamin M, et al. Prevalence of lumbar spinal stenosis, using the diagnostic support tool, and correlated factors in Japan: a population-based study. J Orthop Sci. 2013;18 (6):893-900. doi:10.1007/s00776-013-0455-5

19. Watanabe K, Sekiguchi M, Yonemoto K, et al. DISTO-project working group. Bowel/bladder dysfunction and numbness in the sole of the both feet in lumbar spinal stenosis - a multicenter cross-sectional study. $J$ Orthop Sci. 2017;22(4):647-651. doi:10.1016/j.jos.2017.04.006

20. Shigematsu H, Tanaka M, Kawasaki S, et al. Loco-check presents a useful tool to determine health-related quality of life in elderly people with lumbar spinal stenosis. J Orthop Sci. 2018; pii: S0949-2658(18) 30369-5. doi:10.1016/j.jos.2018.12.001.

21. Park S, Han HS, Kim GU, et al. Relationships among disability, quality of life, and physical fitness in lumbar spinal stenosis: an investigation of Elderly Korean Women. Asian Spine J. 2017;11 (2):256-263. doi:10.4184/asj.2017.11.2.256

22. Haro H, Maekawa S, Hamada Y. Prospective analysis of clinical evaluation and self-assessment by patients after decompression surgery for degenerative lumbar canal stenosis. Spine J. 2008;8(2):380384. doi:10.1016/j.spinee.2007.01.010

23. Gautschi OP, Smoll NR, Joswig H, et al. Influence of age on pain intensity, functional impairment and health-related quality of life before and after surgery for lumbar degenerative disc disease. Clin Neurol Neurosurg. 2016;150:33-39. doi:10.1016/j. clineuro.2016.08.024 